\title{
Knockdown of OLR1 weakens glycolytic metabolism to repress colon cancer cell proliferation and chemoresistance by downregulating SULT2B1 via c-MYC
}

\author{
Tiancheng Zhao ${ }^{1,5}$, Yezhou $\mathrm{Li}^{2,5}$, Kexin Shen ${ }^{3}$, Quan Wang ${ }^{4}$ and Jiayu Zhang $\mathbb{D}^{3 凶}$ \\ (c) The Author(s) 2021
}

Chemoresistance is one of the major problems of colon cancer treatment. In tumors, glycolytic metabolism has been identified to promote cell proliferation and chemoresistance. However, the molecular mechanisms underlying glycolytic metabolism and chemoresistance in colon cancer remains enigmatic. Hence, this research was designed to explore the mechanism underlying the OLR1/C-MYC/SULT2B1 axis in the regulation of glycolytic metabolism, to affect colon cancer cell proliferation and chemoresistance. Colon cancer tissues and LoVo cells were attained, where OLR1, c-MYC, and SULT2B1 expression was detected by immunohistochemistry, RT-qPCR, and western blot analysis. Next, ectopic expression and knockdown assays were implemented in LoVo cells. Cell proliferation was detected by MTS assay and clone formation. Extracellular acidification, glucose uptake, lactate production, ATP/ADP ratio, and GLUT1 and LDHA expression were measured to evaluate glycolytic metabolism. Then, the transfected cells were treated with chemotherapeutic agents to assess drug resistance by MTS experiments and P-gp and SMAD4 expression by RT-qPCR. A nude mouse model of colon cancer transplantation was constructed for in vivo verification. The levels of OLR1, C-MYC, and SULT2B1 were upregulated in colon cancer tissues and cells. Mechanistically, OLR1 increased c-MYC expression to upregulate SULT2B1 in colon cancer cells. Moreover, knockdown of OLR1, c-MYC, or SULT2B1 weakened glycolytic metabolism, proliferation, and chemoresistance of colon cancer cells. In vivo experiments authenticated that OLR1 knockdown repressed the tumorigenesis and chemoresistance in nude mice by downregulating C-MYC and SULT2B1. Conclusively, knockdown of OLR1 might diminish SULT2B1 expression by downregulating C-MYC, thereby restraining glycolytic metabolism to inhibit colon cancer cell proliferation and chemoresistance.

Cell Death and Disease (2022)13:4 ; https://doi.org/10.1038/s41419-021-04174-w

\section{INTRODUCTION}

Colon cancer initiates with an extensive block of cell replication with replication foci shifting towards the upper parts of crypts [1]. Besides, colon cancer is clarified into three discernible stages: initiation, promotion, and progression, where the normal colonic epithelium pathologically transforms into hyperproliferative epitheliums, then into adenomas and carcinoma in situ, and finally into invasive and metastatic cancer [2]. It is well-known that the risk factor for colon cancer includes red meat intake, dietary factors, age, ethnicity, sex, history of colon cancer, enhanced body mass index (BMI), low vegetable and fruit consumption, genetic makeup, low physical activity, and long-term cigarette smoking (30-40 years) [3]. Up to date, patients with colon cancer are generally treated with radiotherapy, cytotoxic drugs, surgery, antiangiogenic agents, and chemotherapy [4]. During treatment, elevated glycolytic metabolism has been observed to assume a pivotal role in adenosine triphosphate (ATP) source in cancer, and ATP-dependent homeostatic maintenance of resting $\left[\mathrm{Ca}^{+}\right]$is crucial in proliferation and chemoresistance of colorectal cancer (CRC) cells [5]. Resistance to chemotherapeutic agents is one of the principal obstacles related to colon cancer treatment, which engenders the need to develop novel therapies' targets [6].

As a lectin-like scavenger receptor, oxidized low-density lipoprotein receptor 1 (OLR1, a gene that encodes for the lectinlike oxidized low-density lipoprotein receptor-1 [LOX-1] protein) recognizes various ligands, including oxidized low-density lipoprotein and has been documented to be involved in cardiovascular and metabolic diseases [7]. Moreover, ectopic expression of OLR1 has been noted to augment colon cancer onset, progression, and metastasis [8]. More importantly, a prior work indicated the promoting effects of OLR1 on gemcitabine resistance in pancreatic cancer [9]. Interestingly, it was illustrated in another research that OLR1 upregulation could enhance c-MYC expression to accelerate pancreatic cancer metastasis [10]. As one of the most critical transcription factors, c-MYC is involved in cancer cells reprogramming, proliferation, and chemoresistance [11]. Existing literature

\footnotetext{
${ }^{1}$ Department of Endoscopy Center, China-Japan Union Hospital of Jilin University, Changchun 130033, P.R. China. ${ }^{2}$ Department of Vascular Surgery, China-Japan Union Hospital of Jilin University, Changchun 130033, P.R. China. ${ }^{3}$ Gastrointestinal Colorectal and Anal Surgery, China-Japan Union Hospital of Jilin University, Changchun 130033, P.R. China.

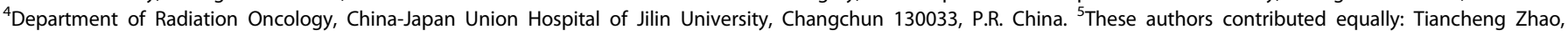
Yezhou Li. ${ }^{凶}$ email: zhangjiayu@jlu.edu.cn Edited by: Dr Jean-Ehrland Ricci
}

Received: 9 May 2021 Revised: 26 August 2021 Accepted: 9 September 2021

Published online: 17 December 2021 
has suggested that ectopically expressed c-MYC facilitates glucose metabolism, viability, and migration of colon cancer cells [12]. Therefore, c-MYC may be implicated in the chemoresistance of colon cancer, which needs to be further verified. Moreover, the overexpression of sulfotransferase (SULT) 2B1 has been exhibited in CRC tissues [13]. Given the aforementioned exploration of literature, we proposed a hypothesis that the network of $O L R 1$, C-MYC, and SULT2B1 might orchestrate metastasis and chemoresistance in colon cancer. Therefore, tissue, cell, and animal assays were implemented in our research to confirm this hypothesis, thus providing newer targets for more effect treatment of colon cancer.

\section{MATERIALS AND METHODS \\ Bioinformatics analysis}

The expression dataset GSE10950 (normal samples: 24 cases; colon cancer samples: 24 cases), GSE41328 (normal samples: 10 cases; colon cancer samples: 10 cases), and GSE75970 (normal samples: 4 cases; colon cancer samples: 4 cases) in Gene Expression Omnibus (GEO, https://www.ncbi. nlm.nih.gov/gds) database was differentially analyzed by "limma" package of $\mathrm{R}$ language (https://bioconductor.org/packages/limma/). The significantly upregulated genes were screened out with $\log \mathrm{FC}>2$ and $p<$ 0.05 as the threshold. Significant upregulated genes were obtained from the intersection of the results of three datasets. Totally, 50 genes most related to colon cancer were obtained from MalaCards (https://www. malacards.org/). The protein interaction network between the significantly upregulated genes and colon cancer-related genes was constructed by String (minimum required interaction score: 0.4 , https://stringdb.org/). The Cytoscape (https://cytoscape.org/) was used to draw protein-protein interaction network and calculate the core degree of significantly upregulated genes.

The Gene Expression Profiling Interactive Analysis (GEPIA, http://gepia2. cancer-pku.cn/\#index) was used to analyze the clinical data (COADREAD) of The Cancer Genome Atlas (TCGA, https://portal.gdc.cancer.gov/) database to determine the impact of key genes on the survival rate of patients with colon cancer. The downstream transcription factors of key genes were identified by literature review. The expression data of key genes and downstream transcription factors in GSE10950, GSE41328, and GSE75970 were normalized. Pearson correlation analysis was used to verify the relationship between key genes and downstream transcription factors. The hTFtarget (http://bioinfo.life.hust.edu.cn/hTFtarget\#!/) was used to predict the downstream genes of transcription factors and obtain important downstream genes by crossing with important significantly upregulated genes. The GEPIA was adopted to analyze the clinical data (COAD, READ) of TCGA database to identify the genes most related to the survival of colon cancer. The Kyoto Encyclopedia of Genes and Genomes (KEGG, https:// www.kegg.jp/kegg/) was used to find the pathways involved in the key downstream genes, and the function of KEGG in colon cancer was predicted based on the literature.

\section{Clinical samples}

From January 2015 to January 2017, 120 patients diagnosed with colon cancer in China-Japan Union Hospital of Jilin University were operated to obtain cancer tissues and normal adjacent tissues. All patients were diagnosed as with colon cancer by pathological diagnosis [14] and did not receive neoadjuvant therapy such as preoperative chemoradiotherapy. Patients meeting the following conditions were excluded from the colon cancer experimental group [1]: advanced cancer with obvious cachexia [2]; multiple tumors [3]; patients with other serious chronic physical diseases, acute infection or liver and kidney failure [4]; patients with mental illness, history of dementia or other reasons cannot communicate; and patients with taking drugs that can cause anxiety and depression.

\section{Immunohistochemical staining}

Paraffin sections were made from colon cancer tissues and adjacent normal tissues. The sections were put into a $60^{\circ} \mathrm{C}$ incubator for $1 \mathrm{~h}$ and taken out. The sections were dewaxed in xylene I and xylene II for $30 \mathrm{~min}$ individually. After the sections were hydrated with gradient alcohol, the sections were put into a beaker containing diluted potassium citrate solution and subjected to $90^{\circ} \mathrm{C}$ microwave antigen thermal repair for $10 \mathrm{~min}$. Then the sections were cooled at room temperature. The endogenous peroxidase was inactivated by adding $3 \% \mathrm{H}_{2} \mathrm{O}_{2}$ in turn at room temperature for $10 \mathrm{~min}$. The sections were sealed at room temperature for $20 \mathrm{~min}$ with $5 \%$ goat serum (Beijing Solarbio Science \& Technology Co. Ltd., Beijing, China). After discarding the blocking solution, the sections were dripped with monoclonal antibodies (Abcam, Cambridge, MA, USA) to LOX-1 (1:100, ab81709) and c-MYC (1:100, ab32072), and polyclonal antibodies (Abcam) to SULT2B1 (1:50, ab254616) and Ki67 $\left(1: 50\right.$, ab15580) to completely cover the sections, and incubated at $4{ }^{\circ} \mathrm{C}$ overnight. Afterwards, the sections were reprobed for $1 \mathrm{~h}$ at $37^{\circ} \mathrm{C}$ with goat-anti-rabbit and anti-mouse secondary antibodies (ZSGB-Bio, Beijing, China). The sections were stained with diaminobenzidine (ZSGB-BIO) for 3-5 min. Multi-functional true-color cell image analysis and management system (Media Cybernetics, Silver Spring, MD, USA) was used for analysis. Totally, four sections were taken from each specimen, and three fields of vision were randomly taken from the sections. The percentage of positive cells was counted under Olympus provis (Olympus, Tokyo, Japan) and the average value was taken to reflect the expression of LOX-1, c-MYC, and SULT2B1, respectively.

\section{Reverse-transcription quantitative polymerase chain reaction (RT-qPCR)}

The Trizol (Invitrogen Inc., Carlsbad, CA, USA) was used to extract total RNA from colon cancer tissues and cells. After RNA concentration was measured by Nanodrop 2000 (Thermo Fisher Scientific Inc., Waltham, MA, USA), $1 \mu \mathrm{g}$ total RNA was reverse transcribed into cDNA by PrimeScript ${ }^{\mathrm{TM}}$ RT reagent kit with gDNA Eraser Kit (Takara Holdings Inc., Kyoto, Japan). After adding $5 \mathrm{~g}$ DNA Eraser Buffer and gDNA Eraser according to the instructions, DNA elimination reaction was carried out at $42^{\circ} \mathrm{C}$ for $2 \mathrm{~min}$. After the reagent was added, the cDNA was obtained by reverse transcription at $37^{\circ} \mathrm{C}$ for $15 \mathrm{~min}$ and $85^{\circ} \mathrm{C}$ for $5 \mathrm{~s}$, and then used for qPCR experiment. Real-time PCR was performed on ABI7500 quantitative PCR instrument (Thermo Fisher Scientific Inc.) with SYBR Premix Ex Taq ${ }^{\text {TM }}$ (Tli RNaseH Plus) Kit (Takara Holdings Inc.). The $2^{-\Delta \Delta C T}$ was used to express the multiple ratio of target gene expression between the observation group and the reference group with glyceraldehyde-3-phosphate dehydrogenase (GAPDH) as internal reference. The formula was as follows: $\Delta \triangle C T=\Delta C T$ observation group -

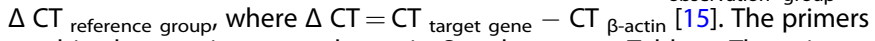
used in the reaction were shown in Supplementary Table 1. The primers were provided by Shanghai GenePharma Co. Ltd. (Shanghai, China). The experiment was repeated three times.

\section{Western blot analysis}

The Bicinchoninic Acid Kit (Thermo Fisher Scientific Inc.) was used to detect the protein concentration in colon tumor tissues of nude mice. The $30 \mu \mathrm{g}$ of total protein was electrophoresed by sodium dodecyl sulfate polyacrylamide gel electrophoresis with constant voltage of $80 \mathrm{~V}$ for $35 \mathrm{~min}$, followed by $120 \mathrm{~V}$ for $45 \mathrm{~min}$. After electrophoresis, it was transferred to a polyvinylidene fluoride membrane (Amersham Biosciences/GE Healthcare, Piscataway, NJ, USA). The membrane was sealed at room temperature for $1 \mathrm{~h}$ with $5 \%$ skimmed milk powder. After dumping the sealing fluid, the membrane was probed overnight with antibodies (Abcam) to LOX-1 monoclonal antibody (1:1000, ab214427), c-MYC monoclonal antibody (1:1000, ab32072), SULT2B1 polyclonal antibody (1:500, ab254616), and $\beta$-actin monoclonal antibody (1:5000, ab8225) at $4{ }^{\circ} \mathrm{C}$. The membrane was washed with Tris-buffered saline with Tween 20 (TBST) buffer (Tris-buffered saline buffer containing $0.1 \%$ Tween-20) for 3 times/10 min. Afterwards, the membrane was reprobed for $1 \mathrm{~h}$ with horseradish peroxidase (HRP)-tagged goat-anti-mouse or anti-rabbit (1:10,000, 115-035-003; Jackson Laboratory, Bar Harbor, ME, USA) secondary antibodies at room temperature. The membrane was washed with TBST for 3 times/10 min. After scanning and developing by optical luminescent instrument (General Electric Company, Schenectady, NY, USA), the gray analysis of protein bands was carried out using Image Pro Plus 6.0 software (Media Cybernetics, Silver Spring, MD, USA), and the relative expression of other proteins in corresponding samples was corrected with the amount of $\beta$-actin protein as internal reference. The experiment was repeated three times.

\section{Cell culture and lentivirus transfection}

Human colon cancer LoVo cell line was purchased from American Type Culture Collection (ATCC, VA, USA) and cultured in $37^{\circ} \mathrm{C}, 5 \% \mathrm{CO}_{2}$ incubator. The OLR1 knockdown lentivirus, c-MYC overexpression lentivirus, c-MYC knockdown lentivirus, SULT2B1 overexpression lentivirus, and blank vector lentivirus were purchased from Shanghai 
Genechem Co., Ltd. (Shanghai, China). The cells were infected according to the lentivirus infection manuals provided by Shanghai Genechem Co., Ltd. According to the virus titer multiplicity of infection $(\mathrm{MOI})=5$, appropriate amount of lentivirus was added into the cell culture plate. After $48 \mathrm{~h}$ of culture, the puromycin (60210ES25, Yeasen Company, Shanghai, China) was added to screen stable cell lines. The expression of OLR1, C-MYC, and SULT2B1 in each cell line was detected by RT-qPCR to ensure effective cell lines.

The LoVo cells were infected with lentiviruses harboring: (1) short hairpin (sh)-negative control (NC) or sh-OLR1; (2) overexpression (oe)$\mathrm{NC}$, oe-c-MYC, sh-NC, or sh-c-MYC; (3) sh-NC + oe-NC, sh-OLR1 + oe-NC, or sh-OLR1 + oe-C-MYC; (4) sh-NC + oe-NC, sh-C-MYC + oe-NC, or sh-CMYC + oe-SULT2B1; (5) sh-NC + oe-NC, sh-OLR1 + oe-NC, or sh-OLR1 + oe-SULT2B1.

\section{MTS experiment}

Colon cancer cells in logarithmic growth phase were digested with trypsin to prepare $3 \times 10^{5}$ cells $/ \mathrm{mL}$ cell suspension. The $100 \mu \mathrm{L}$ cell suspension was added to each well of a 96-well plate. After the cells adhered to the wall, cells were added with $100 \mu \mathrm{L}$ chemotherapeutic drugs prepared by culture medium containing 10\% serum [oxaliplatin (O9512-5MG; the concentration gradients of $0,2,4,8,16$, and $32 \mathrm{mg} / \mathrm{L}), 5$-fluorouracil (5-Fu, F5130100MG; the concentration gradients of $0,2.5,5,10,20$ and $40 \mathrm{mg} / \mathrm{L}$ ), cisplatin (DDP, P4394-25MG; the concentration gradients of $0,0.5,1,2,4$, and $8 \mathrm{mg} / \mathrm{L})$, paclitaxel $(444375-500 \mathrm{MG}$; the concentration gradients of 0 , $12.5,25,50,100$, and $200 \mathrm{mg} / \mathrm{L}$ ), the above drugs were purchased from Sigma-Aldrich Chemical Company (St Louis, MO, USA)] [16]. After $72 \mathrm{~h}$ of administration, $20 \mu \mathrm{L}$ of MTS reagent (G1111; Promega Corporation, Madison, WI, USA) was added to each well and incubated in a $37^{\circ} \mathrm{C}$ and $5 \% \mathrm{CO}_{2}$ incubator for $4 \mathrm{~h}$. The absorbance $(A)$ at $490 \mathrm{~nm}$ (A490) of each well was detected on the multi-functional microplate reader. The survival rate and inhibition rate were calculated by the absorbance value. According to the linear regression equation of drug concentration and inhibition rate, the $\mathrm{IC}_{50}$ value of drug inhibition rate was determined. Cell viability curve was drawn with concentration as abscissa and OD value as ordinate. In addition, the culture plates were taken out severally after $12 \mathrm{~h}$, 24,36 , and $48 \mathrm{~h}$ of culture. The $20 \mu \mathrm{L}$ MTS reagent was added to each well, and the cell proliferation level was detected after $4 \mathrm{~h}$ of culture. Cell viability curve was drawn with time point as abscissa and OD value as ordinate. The experiment was repeated three times.

\section{Clone formation experiment}

Colon cancer cells in logarithmic growth phase were digested with $0.25 \%$ trypsin to prepare single cell suspension. After cell counting, 1000 cells were added into each well of a 12-well plate and shaken evenly. The cells were cultured in the $37^{\circ} \mathrm{C}, 5 \% \mathrm{CO}_{2}$ incubator, and the medium was changed every 2 days. When more than 50 cell clones were observed under microscope (about 7-14 days), the experiment was terminated. The cells were fixed with $4 \%$ polyformaldehyde for $15 \mathrm{~min}$, and stained with $1 \%$ crystal violet (C0121; Beyotime Biotechnology Co., Shanghai, China) for $10 \mathrm{~min}$. After the crystal violet staining solution was poured out, the staining solution of the cells was slowly washed away with running water and dried in the air. The 12-well plate were photographed under the microscope (Olympus, Tokyo, Japan) and the number of clones per well was counted. The experiment was repeated three times.

\section{Glucose uptake test}

The colon cancer cells infected with sh-NC + oe-NC, sh-c-MYC + oe-NC, shc-MYC + oe-SULT2B1, sh-OLR1 + oe-NC, or sh-OLR1 + oe-SULT2B1 were added into the 6 -well plate with $1 \times 10^{5}$ cells per well. After $24 \mathrm{~h}$ in serumfree medium, cell culture medium was collected and centrifuged at room temperature for $5 \mathrm{~min}$. After removing the precipitates, the supernatant was taken out. The blank and standard controls were set. According to the method of glucose determination kit (BC2505, Beijing Solarbio Science \& Technology Co. Ltd.), enzyme colorimetry was used for determination. The working solution was prepared according to the ratio of R1 reagent to R2 reagent of $1: 1$, and used after mixing evenly. The $10 \mu \mathrm{L}$ distilled water, calibration solution, and supernatant was added into $1000 \mu \mathrm{L}$ working solution, respectively, to configure blank tube, calibration tube, and sample tube. After adding the sample, it was mixed well with vortex oscillator, placed in $37^{\circ} \mathrm{C}$ constant temperature water bath, and reacted in water bath for $10 \mathrm{~min}$. The absorbance value of the final product was detected at $505 \mathrm{~nm}$ by enzyme reader (BioTek, VT, USA). According to the instructions of the kit, the glucose level in the supernatant was calculated, which was the glucose uptake of the cells. The experiment was repeated three times.

\section{Lactic acid production test}

According to the method of lactic acid determination kit (93-K627-100, BioVision, San Francisco, CA, USA), the standard curve was established with lactate standard. The mixture of $20 \mu \mathrm{L}$ supernatant, $26 \mu \mathrm{L}$ buffer, and $2 \mu \mathrm{L}$ lactate enzyme was absorbed, mixed evenly, and placed at room temperature for $30 \mathrm{~min}$. The absorbance of the final product at $570 \mathrm{~nm}$ was detected by microplate reader (BioTek). According to the standard curve, the level of lactic acid in the supernatant was calculated, which was the amount of lactic acid produced by cells. The experiment was repeated three times.

\section{Determination of ATP (adenosine triphosphate)/ADP (adenosine diphosphate) ratio by high-performance liquid chromatography (HPLC)}

The colon cancer cells infected with sh-NC + oe-NC, sh-c-MYC + oe-NC, shc-MYC + oe-SULT2B1, sh-OLR1 + oe-NC, or sh-OLR1 + oe-SULT2B1 were cultured in $150 \mathrm{~mL}$ culture bottle with $1 \times 10^{6}$ conventional cells. After $24 \mathrm{~h}$ of culture, the cell culture medium was collected. After centrifugation for $5 \mathrm{~m}$, the cells were centrifuged repeatedly and rinsed once and counted. The cytomembrane was broken with $500 \mu \mathrm{L}$ of $0.1 \mathrm{mmol} / \mathrm{L}$ perchloric acid. After centrifugation for $20 \mathrm{~min}$, the supernatant was $250 \mu \mathrm{L}$. The $50 \mu \mathrm{L}$ of $0.5 \mathrm{mmol} / \mathrm{L} \mathrm{Na}_{2} \mathrm{CO}_{3}$ was added to mix. After centrifugation for $10 \mathrm{~m}, 50 \mu \mathrm{L}$ supernatant was taken out to leave it for machine detection. HPLC detection method: ATP or ADP standard was taken to prepare $1 \mathrm{nmol} / \mathrm{L}$ solution. Afterwards, it added $\mathrm{ddH}_{2} \mathrm{O}$ to make a constant volume of $50 \mu \mathrm{L}$ and chromatographic loading of $20 \mu \mathrm{L}$. The content of the sample was calculated by comparing the elution peak of the standard. ATP and ADP contents were expressed in $\mathrm{nmol} / 10^{6}$ cells. Analysis conditions of HPLC: Waters 510 Pump Hypersil C18 $(250 \mathrm{~mm} \times 4.6 \mathrm{~mm}$, the particle size of packing is $5 \mu \mathrm{M}$ ), column temperature $25^{\circ} \mathrm{C}$, the mobile phase was $0.1 \mathrm{mmol} / \mathrm{L} \mathrm{KH} 2 \mathrm{PO} 4(\mathrm{pH} 6.0$ ), the injection volume was $20 \mu \mathrm{L}$, pump flow rate was $0.9 \mathrm{~mL} / \mathrm{min}$, constant speed elution, Waters 484 Photodiode Array Detector, the UV detection wavelength was $254 \mathrm{~nm}$, Baseline 810 chromatographic workstation.

\section{Seahorse experiment}

The Seahorse XF96 flux analyzer (Seahorse Bioscience, Billerica, MA, USA) was used to detect the extracellular acidification rate (ECAR) reflecting the activity of glycolytic metabolism. The cells were laid $24 \mathrm{~h}$ in advance. Colon cancer cells were seeded into the Seahorse XF cell culture plate at a density of $1 \times 10^{4}$ per well and cultured overnight in growth medium. The instrument was preheated, seahorse instrument and computer were turned on, and software was turned on to make the instrument temperature rise to $37^{\circ} \mathrm{C}$ with overnight preheating and hydration probe. The Seahorse XF calibration solution was added into the Utility Plate. The test plate was put back on the Utility Plate. And it was placed in a $\mathrm{CO}_{2}$-free incubator at $37^{\circ} \mathrm{C}$ overnight to hydrate the probe. Drug concentration required for cell glycolysis pressure test (103020-100, Seahorse Bioscience): $10 \mathrm{mmol} / \mathrm{L}$ glucose, $1 \mathrm{~mol} / \mathrm{L}$ oligomycin, and $50 \mathrm{mmol} / \mathrm{L}$ 2-deoxyglucose (2DG). Mitochondrial stress test kit (103015-100, seahorse Bioscience), 1 mol/ L oligomycin, $1 \mathrm{~mol} / \mathrm{L}$ carbonyl cyanide 4-(trifluoromethoxy)phenylhydrazone (FCCP), $0.5 \mathrm{~mol} / \mathrm{L}$ rotenone, and $0.5 \mathrm{~mol} / \mathrm{L}$ actinomycin $A$. Both assays were normalized by total protein quantification. The experiment was repeated three times.

\section{Establishment and grouping of colon cancer xenograft model in nude mice}

A total of 70 nude mice were purchased from the experimental animal center of the Third Military Medical University. The BALB/c nude mice aged 4 weeks and specific-pathogen-free (SPF) were fed on laminar flow shelves under the conditions of constant temperature $\left(24-26^{\circ} \mathrm{C}\right)$ and constant humidity $(45-55 \%)$. Feed and drinking water were used after hightemperature disinfection.

The colon cancer cells in logarithmic growth phase were prepared into suspension containing $1 \times 10^{6}$ colon cancer cells $(200 \mu \mathrm{L})$. lodophor solution was used to disinfect the skin of inoculation site. The $200 \mu \mathrm{L}$ single cell suspension was subcutaneously injected into the left armpit of nude mice with a $1 \mathrm{~mL}$ syringe. After implantation, the nude mice were kept in SPF environment. Tumor nodules appeared in the inoculation site 
A

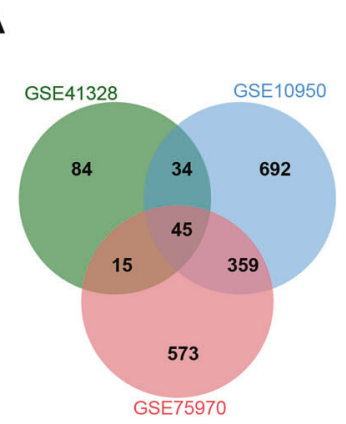

D

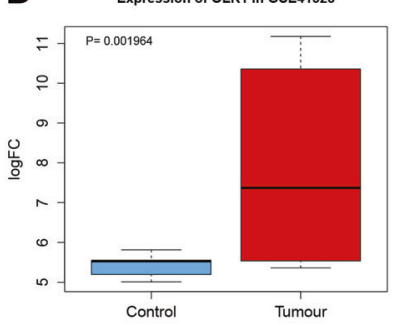

H

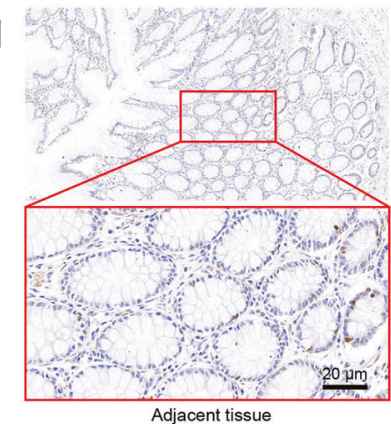

B

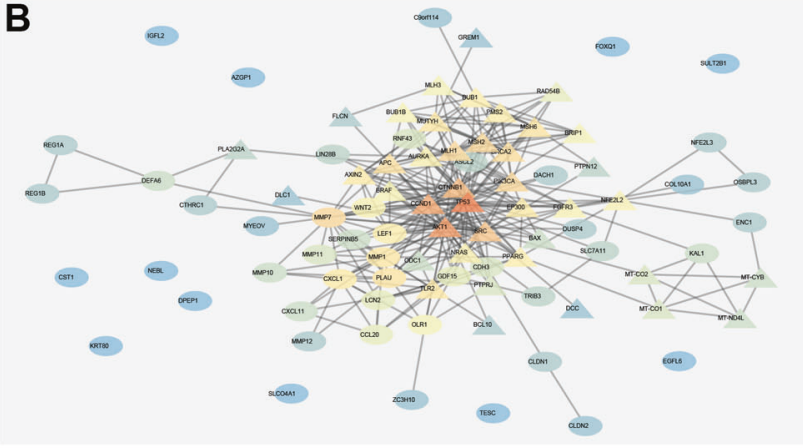

E
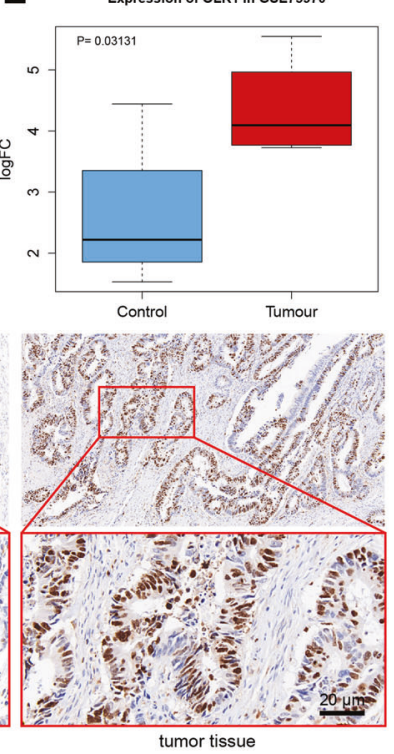

F

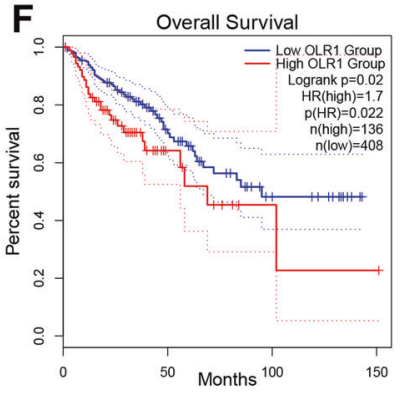

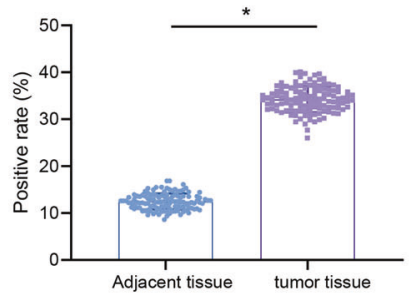

C

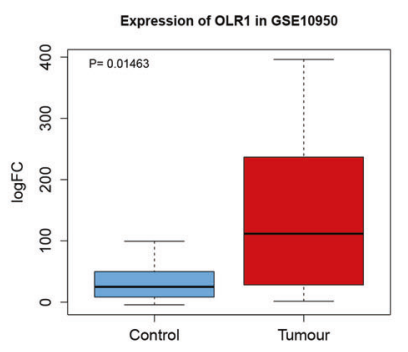

G
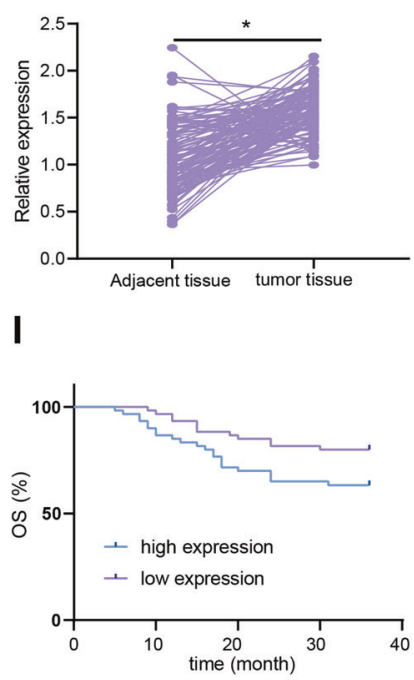

Fig. 1 OLR1 expression is high in colon cancer tissues. A Venn map of upregulated genes in GSE10950, GSE41328, and GSE75970, which obtained 45 overlapping genes. B Protein interaction network of 45 overlapping genes and 50 important genes of colon cancer from MalaCards. Circles represented intersecting genes and triangles represented genes obtained by MalaCards. The redder the color of the gene image, the higher the degree of core. Conversely, the bluer the color, the lower the degree of core. C The box diagram of the expression of OLR1 in GSE10950 (control $n=24$; tumor $n=24$ ). D The box diagram of the expression of OLR1 in GSE41328 (control $n=24$; tumor $n=24)$. E The box diagram of the expression of OLR1 in GSE75970 (control $n=24$; tumor $n=24$ ). The blue box indicated the expression of normal samples, and the red box indicated the expression of tumor samples. F GEPIA to analyze TCGA clinical data to draw the curve between OLR1 expression and colon cancer survival. G RT-qPCR to detect the expression of OLR1 mRNA in colon cancer tissues and adjacent normal tissues (adjacent tissues: $n=120$; tumor tissues: $n=120$ ). $\mathbf{H}$ The expression of LOX-1 protein in colon cancer tissues and adjacent normal tissues detected by immunohistochemistry (adjacent tissues: $n=120$; tumor tissues: $n=120 ; \times 500$ ). I Kaplan-Meier curve of the relationship between OLR1 and survival in patients with colon cancer. ${ }^{*} p<0.05$ between two groups.

of nude mice about 2 weeks, and the texture was hard. When the tumor diameter was about $7 \mathrm{~mm}$, it was considered that the nude mice model of colon cancer transplantation was successful.

Oxaliplatin intraperitoneal injection was performed [17]. The total dosage of oxaliplatin was calculated according to $30 \mathrm{mg} / \mathrm{g}$ body weight, and was given in five times. The dosage of each injection was $6 \mathrm{mg} / \mathrm{kg}$, once every 3 days by intraperitoneal injection, five times in a row. Since the first administration, the maximum long diameter and vertical short diameter $(\mathrm{mm})$ of tumor were measured by vernier caliper, which were expressed as $a$ and $b$, respectively. The tumor volume $(V)$ was calculated according to the following formula: $V=a \times b^{2} \times 0.5$ [18]. The tumor volume was measured every 5 days and four times in a row to draw tumor growth curve of tumor-bearing mice. The nude mice were euthanized after drug withdrawal one week. The tumor was dissected, weighed and the inhibition rate was calculated as [(average tumor weight of control group - average tumor weight of treatment group)/average tumor weight of control group $\times 100 \%]$ [18].

Nude mice were subcutaneously inoculated with colon cancer cells infected with sh-NC + oe-NC, sh-OLR1 + oe-NC, or sh-OLR1 + oe-SULT2B1; or [2] subcutaneously inoculated with colon cancer cells infected with shNC or sh-OLR1 and then subcutaneously injected with PBS or oxaliplatin ( $n=10$ mice/group).

\section{Statistical analysis}

SPSS21.0 (IBM-SPSS Inc., Chicago, IL, USA) was implemented for statistical analysis. The measurement data were expressed in the form of mean \pm standard deviation. The enumeration data were indicated as a rate or percentage. The $x 2$ test was used in the comparison of enumeration data. Independent sample $t$-test was adopted for comparison between the two groups. One-way analysis of variance (ANOVA) was applied for comparison among multiple groups. Kaplan-Meier survival method and Log-rank test were used to analyze the relationship between expression intensity of OLR1 and survival time of patients with colon cancer. $p<0.05$ was considered to be statistically significant difference.

\section{RESULTS}

OLR1 is negatively correlated with the survival of patients with colon cancer

In order to find the targets for the treatment and diagnosis of colon cancer, 1130, 178, and 992 upregulated genes in colon cancer were found by differential analysis of expression datasets GSE10950, GSE41328, and GSE75970, respectively, which obtained 45 genes from their intersection (Fig. 1A). The 50 colon cancer-related genes 
A
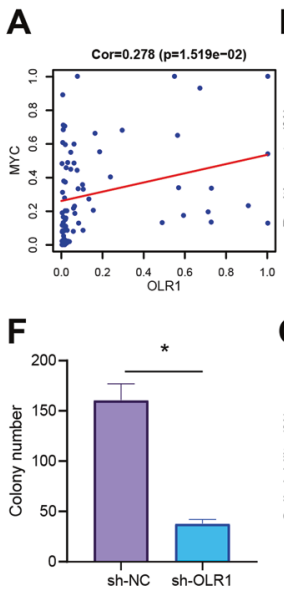

B
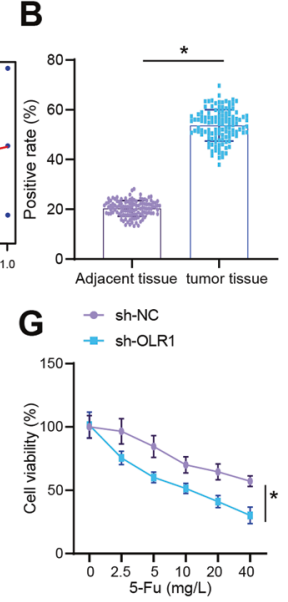

C

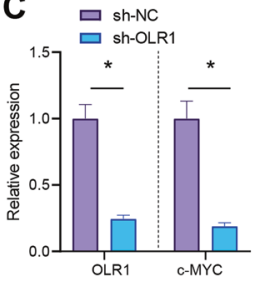

- sh-NC

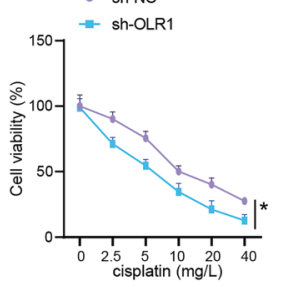

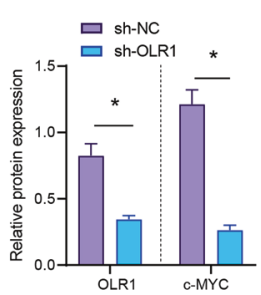

- sh-NC

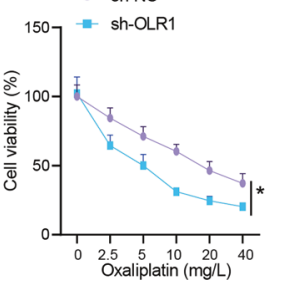

D

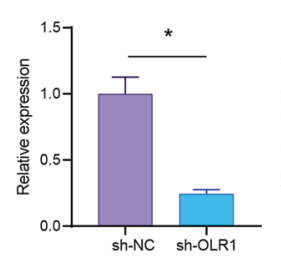

- sh-NC

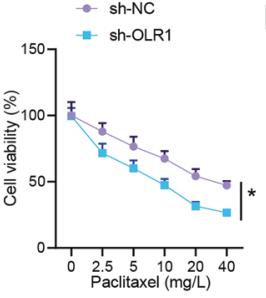

E $\rightarrow$ sh-Nc

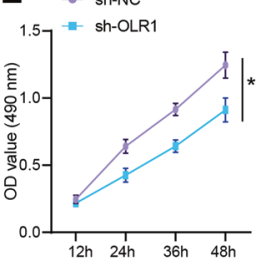

H $\square$ sh-NC

H $\square$ sh-OLR1

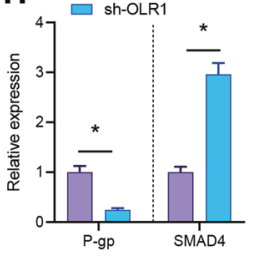

Fig. 2 OLR1 knockdown depresses c-MYC expression and the proliferation and chemoresistance of colon cancer cells. A Correlation map of expression data of OLR1 and c-MYC in GSE10950, GSE41328, and GSE75970. B Immunohistochemical staining to detect the expression of c-MYC protein in colon cancer tissues and adjacent normal tissues (adjacent tissues: $n=120$; tumor tissues: $n=120$ ). C RT-qPCR and western blot analysis to detect the expression of OLR1 and c-MYC in colon cancer cells. D Detection of Ki67 mRNA expression in colon cancer cells by RT-qPCR. E MTS to detect the proliferation of colon cancer cells. F Colony formation assay to detect the colony formation of colon cancer cells. G The drug resistance of colon cancer cells to DDP, oxaliplatin, 5-Fu, and paclitaxel detected by MTS. H RT-qPCR to detect the mRNA expression of P-gp and SMAD4 in colon cancer cells. The cell experiment was repeated three times. ${ }^{*} p<0.05$ between two groups.

were obtained from MalaCards, and the protein-protein interaction network of these 95 genes was constructed using String (Fig. 1B) to calculate the core degree of 45 significantly upregulated genes. Thus, the top 10 genes were retrieved, among which the research on OLR1 was the least. Moreover, OLR1 was highly expressed in colon cancer in these three datasets (Fig. 1C-E). The results suggested that OLR1 might be involved in the occurrence and development of colon cancer.

In order to further determine the role of $O L R 1$ in colon cancer, the clinical data (COADREAD) of colon cancer in the TCGA were analyzed by GEPIA. The results displayed that the survival rate of colon cancer patients with high expression of OLR1 was strikingly decreased (Fig. 1F). As reflected by RT-qPCR and immunohistochemical staining in 120 cases of colon cancer tissues and adjacent normal tissues, OLR1 expression was potently higher in colon cancer tissues than that in adjacent normal tissues, and LOX-1 protein was mainly located in the cell membrane of colon cancer tissues (Fig. 1G, H).

As documented in Supplementary Table 2, high expression of OLR1 in colon cancer tissues had sharp positive correlation with lymph node metastasis and tumor infiltration depth of colon cancer patients, whereas the relative expression of OLR1 was not correlated with age, gender, tumor size, tumor differentiation degree, and tumor location of patients. Based on the results of Kaplan-Meier, the overall survival of colon cancer patients with $O L R 1$ low expression was dramatically longer than that in patients with high expression (Fig. 1I), which was consistent with the biological prediction. In brief, OLR1 was highly expressed in colon cancer tissues, and was negatively correlated with survival time of colon cancer patients, and positively correlated with lymph node metastasis and tumor infiltration depth of colon cancer patients.

\section{Knockdown of OLR1 downregulates C-MYC and inhibits the proliferation and chemoresistance of colon cancer cells}

In order to further determine the relationship between OLR1 and C-MYC in colon cancer, the expression data of OLR1 and c-MYC in GSE10950, GSE41328, and GSE75970 were normalized, respectively. Then the expression correlation between OLR1 and c-MYC was analyzed by merging these three datasets. The results showed that OLR1 expression was positively correlated with c-MYC in colon cancer (Fig. 2A), indicting OLR1 might promote c-MYC expression in colon cancer. Immunohistochemistry authenticated that compared with adjacent normal tissues, c-MYC was substantially upregulated in colon cancer tissues, which was mainly located in the nucleus of colon cancer tissues (Fig. 2B).

The OLR1 stably knockdown cell lines and control cell lines were constructed to explore the regulation of c-MYC expression by $O L R 1$ in colon cancer cells and its effect on the function of colon cancer cells. As for the result of RT-qPCR and western blot analysis, knockdown of OLR1 evidently reduced the transcription level of C-MYC (Fig. 2C). In addition, according to RT-qPCR result, knockdown of OLR1 strikingly disturbed proliferation marker Ki67 expression (Fig. 2D). Meanwhile, MTS test and clone formation test exhibited knockdown of OLR1 remarkably decreased the proliferation and clone formation of colon cancer cells (Fig. 2E, F).

The sensitivity of LoVo colon cancer cells to chemotherapeutic drugs and P-gp and SMAD4 expression [19] were detected before and after the knockdown of $O L R 1$. In the experiment of cell drug resistance, the conventional treatment drugs such as DDP, oxaliplatin, 5-Fu, and paclitaxel were selected for the study [16]. The results documented that the sensitivity of LoVo colon cancer cells to DDP, oxaliplatin, 5-Fu, and paclitaxel was obviously increased, P-gp expression was significantly decreased, and SMAD4 expression was clearly enhanced after knocking down OLR1 (Fig. 2G, H). In conclusion, high expression of c-MYC in colon cancer tissues and cells was observed, and knockdown of OLR1 could downregulate c-MYC and repress the proliferation and chemoresistance of colon cancer cells.

\section{Knockdown OLR1 suppresses the proliferation and chemoresistance of colon cancer cells by downregulating c-MYC}

In order to further verify that $O L R 1$ regulated the proliferation and chemoresistance of colon cancer cells by promoting c-MYC, OLR1 was knocked down and/or c-MYC was overexpressed in colon cancer cells. From RT-qPCR result, OLR1 and c-MYC mRNA expression was obviously diminished in OLR1-silenced colon cancer cells, while in the presence of sh-OLR1, elevated c-MYC mRNA expression was observed in colon cancer cells after further oe-c-MYC treatment (Fig. 3A). As detected by MTS assay, colony formation assay, and RT-qPCR, the proliferation, colony formation, and Ki67 expression of LoVo colon cancer cells was apparently 


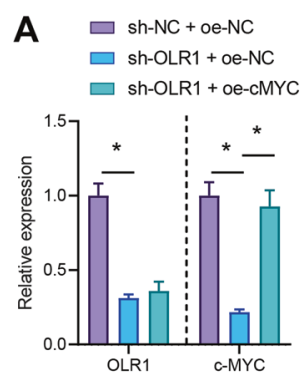

E

- sh-NC + oe-NC

-- sh-OLR1 + oe-NC

- sh-OLR1 + oe-cMYC

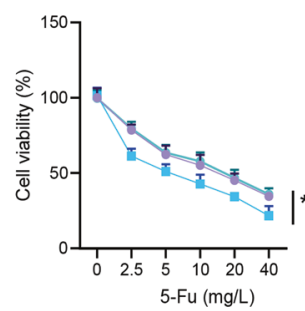

B

$$
\begin{aligned}
& - \text { sh-NC + oe-NC } \\
& - \text { sh-OLR1 + oe-NC } \\
& + \text { sh-OLR1 + oe-cMYC }
\end{aligned}
$$

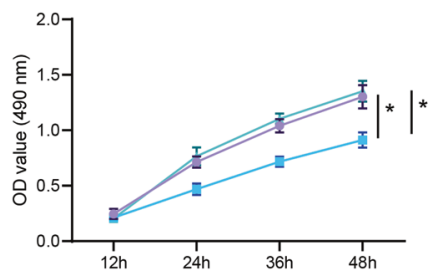

$$
\text { - sh-NC + oe-NC }
$$$$
\text { - sh-OLR1 + oe-NC }
$$$$
\text { ^- sh-OLR1 + oe-cMYC }
$$

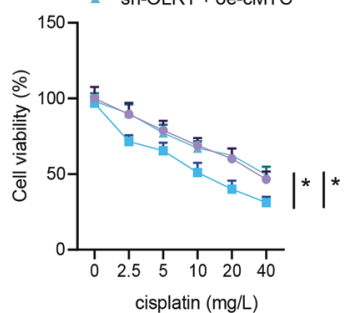

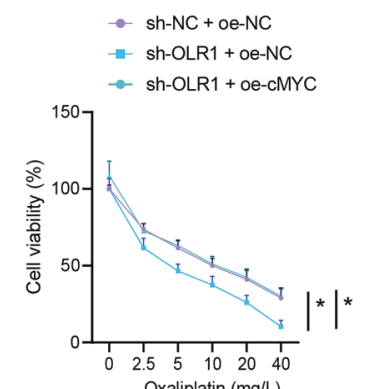

Oxaliplatin ( $\mathrm{mg} / \mathrm{L})$

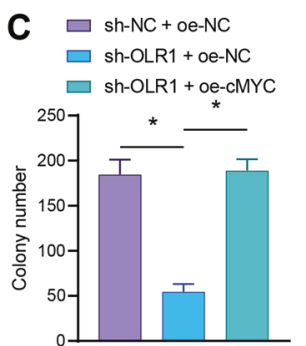
D $\square$ sh-NC + oe-NC $\square$ sh-OLR1 + oe-NC $\square$ sh-OLR1 + oe-cMYC

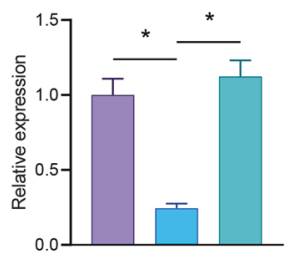

F $\square$ sh-NC + oe-NC

- sh-NC + oe-NC

- sh-OLR1 + oe-NC

4 sh-OLR1 + oe-cMYC

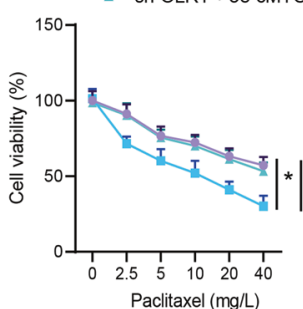

OLR1 + oe-NC

sh-OLR1 + oe-cMYC

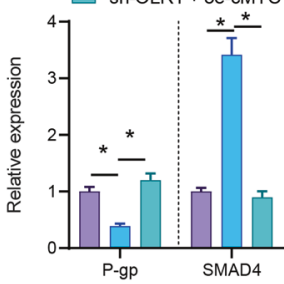

Fig. 3 OLR1 knockdown causes c-MYC downregulation to restrain proliferation and chemoresistance of colon cancer cells. Colon cancer cells were treated with sh-NC + oe-NC, sh-OLR1 + oe-NC, or sh-OLR1 + oe-c-MYC. A The mRNA expression of OLR1 and c-MYC in colon cancer cells determined by RT-qPCR. B MTS to measure the proliferation of colon cancer cells. C Colony formation of colon cancer cells detected by colony formation assay. D The mRNA expression of Ki67 in colon cancer cells checked by RT-qPCR. Colon cancer cells were treated with sh-NC + oe-NC, sh-OLR1 + oe-NC, or sh-OLR1 + oe-c-MYC, followed by treatment with DDP, oxaliplatin, 5-Fu, and paclitaxel. E The drug resistance of colon cancer cells to DDP, oxaliplatin, 5-Fu, and paclitaxel assessed by MTS. F RT-qPCR to detect the mRNA expression of P-gp and SMAD4 in colon cancer cells. The cell experiments were repeated three times. ${ }^{*} p<0.05$ between two groups.

decreased by sh-OLR1 treatment, which was abrogated by further oe-c-MYC treatment (Fig. 3B-D). Furthermore, the sensitivity of LoVo colon cancer cells to DDP, oxaliplatin, 5-Fu, and paclitaxel was distinctly enhanced, P-gp expression was authentically declined, and SMAD4 expression was noticeably augmented subsequent to OLR1 knockdown, which was reversed by further overexpressing c-MYC (Fig. 3E, F). Collectively, knockdown of OLR1 could inhibit the proliferation and chemoresistance of colon cancer cells via c-MYC downregulation.

\section{Knockdown of C-MYC reduces SULT2B1 expression and inhibits the glycolytic metabolism of colon cancer cells} Next, totally 14,161 downstream genes of c-MYC were predicted by hTFtarget and intersected with 45 significantly upregulated genes, which found that 12 genes were critical downstream genes of c-MYC (Fig. 4A). By analyzing the clinical data (COADREAD) of colon cancer in TCGA database through GEPIA, the relationship between these 12 genes and survival of colon cancer was obtained, which found that only SULT2B1 and GDF15 were certainly correlated with survival, and the correlation of SULT2B1 was significantly higher than that of GDF15 (Supplementary Table 3 and Fig. 4B). Therefore, we believed that SULT2B1 might be a key downstream gene of c-MYC in colon cancer. The results of immunohistochemical staining exhibited that compared with the adjacent normal tissues, SULT2B1 was highly expressed in colon cancer tissues and mainly located in the cytoplasm of colon cancer tissues (Fig. 4C).

Furthermore, the colon cancer cells were infected with lentivirus to confirm that C-MYC could orchestrate SULT2B1 expression. As reflected by RT-qPCR, increased c-MYC and SULT2B1 expression in LoVo colon cancer cells was noticed following C-MYC overexpression, which was opposite after silencing c-MYC (Fig. 4D), indicating that c-MYC could upregulate SULT2B1.

It was found that SULT2B1 was related to the steroid hormone biosynthesis (hsa00140) pathway using KEGG database [20]. Through literature review, we found that steroid hormones could mediate glycolytic metabolism that could impact cell proliferation and chemoresistance of various cancers [21-25]. Therefore, ECAR, glucose uptake, lactate production, ATP/ADP ratio, and the expression of glycolysis-related genes GLUT1 and LDHA [26] were detected. On the basis of detection result, the ECAR, glucose intake, lactate production, ATP/ADP ratio, and GLUT1 and LDHA expression of LoVo colon cancer were apparently diminished by knocking down c-MYC (Fig. 4E-l). The results suggested that knockdown of c-MYC could inhibit glycolytic metabolism of colon cancer cells. Taken together, SULT2B1 was highly expressed in colon cancer cells, and knockdown of c-MYC could disturb SULT2B1 expression and inhibit the glycolytic metabolism of colon cancer cells.

Knockdown of c-MYC inhibits the proliferation and chemoresistance of colon cancer cells by decreasing SULT2B1 expression to restrain glycolytic metabolism

In order to further explore whether c-MYC manipulated glycolytic metabolism through SULT2B1 to affect the proliferation and chemoresistance of colon cancer cells, we successfully constructed a cell line with c-MYC knockdown and SULT2B1 overexpression (Fig. 5A). The ECAR, glucose uptake, lactate production, ATP/ADP ratio, and GLUT1 and LDHA (glycolysis-related genes) expression were detected, which displayed that overexpression of SULT2B1 restored the inhibition of glycolysis induced by c-MYC knockdown (Fig. 5B-F). Meanwhile, MTS assay, clone formation assay, and RTqPCR manifested that overexpression of SULT2B1 negated the repressive effect of c-MYC knockdown on colon cancer cell proliferation (Fig. 5G-I).

In addition, control cells (the sh-NC + oe-NC group), c-MYC knockdown cells (the sh-c-MYC + oe-NC group), and c-MYC knockdown cells with overexpression of SULT2B1 (the sh-c-MYC + oeSULT2B1 group) were stimulated with chemotherapeutic drugs including DDP, oxaliplatin, 5-Fu, and paclitaxel. Overexpression of SULT2B1 neutralized the sensitization effect of colon cancer cells to chemotherapeutic drugs induced by c-MYC knockdown (Fig. 5J, K). In short, knockdown of c-MYC could decline SULT2B1 expression to 
A

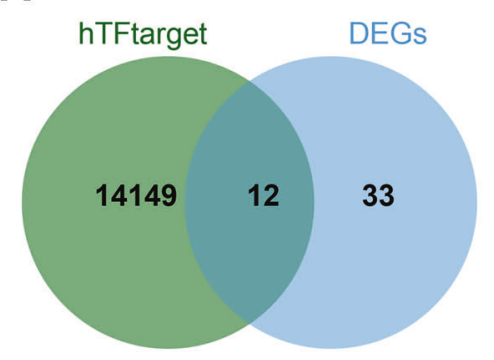

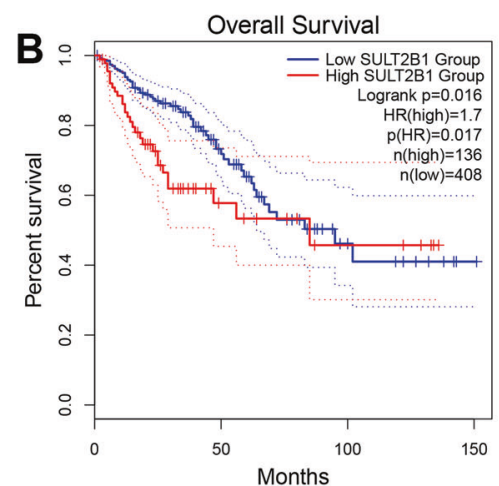

C

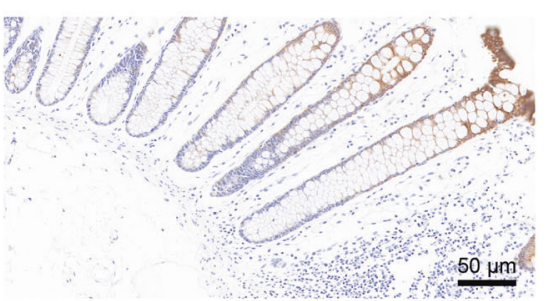
Adjacent tissue

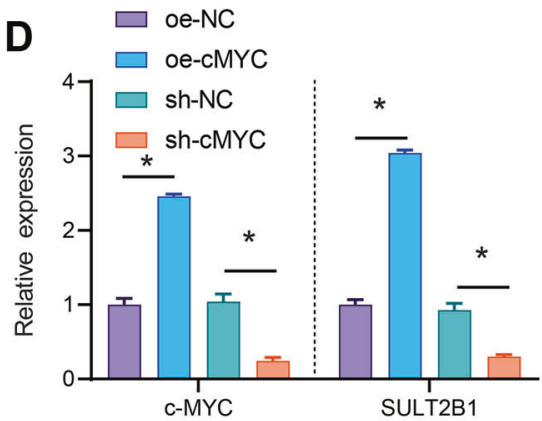

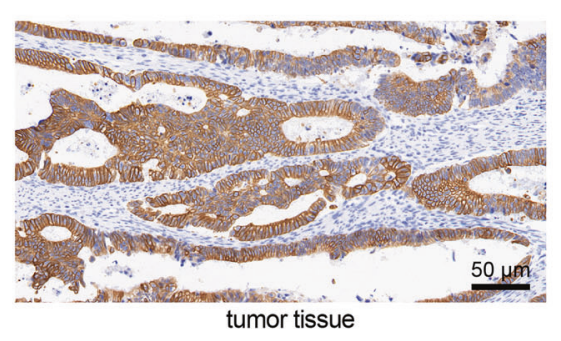

E

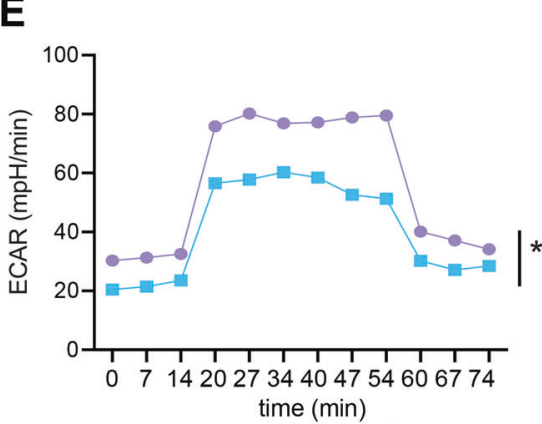

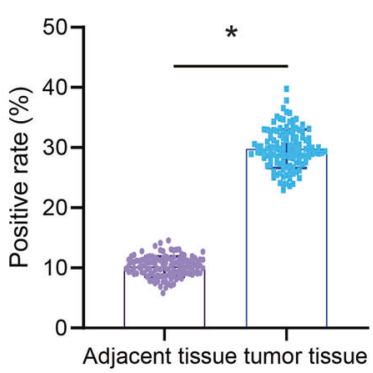

F

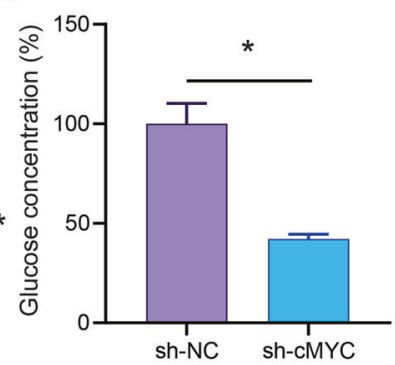

G

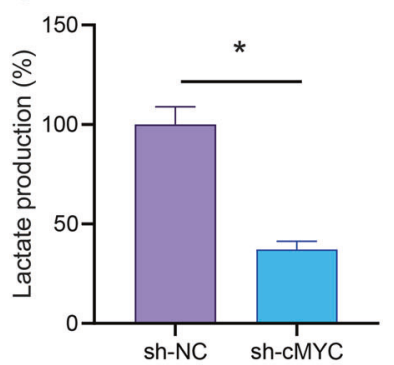

H

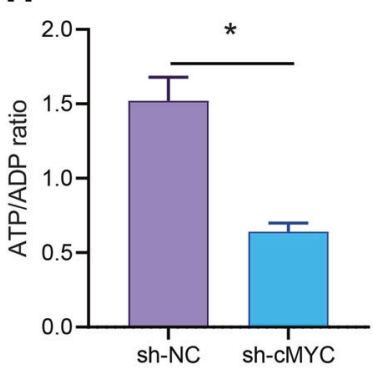

I

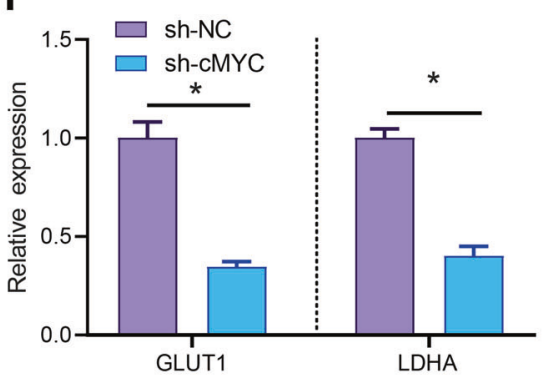

Fig. 4 c-MYC knockdown diminishes SULT2B1 expression and dampened glycolytic metabolism of colon cancer cells. A The downstream genes of transcription factor c-MYC predicted by hTFtarget and intersected with 45 obviously upregulated genes to obtain 12 genes. B Clinical data of TCGA analyzed by GEPIA to draw the survival curve between SULT2B1 expression and colon cancer. C The protein expression of c-MYC in colon cancer and adjacent normal tissues detected by immunohistochemistry (adjacent tissues: $n=120$; tumor tissues: $n=120$; $\times 200$ ). D The mRNA expression of c-MYC and SULT2B1 in colon cancer cells after overexpression and knocking down c-MYC determined by RT-qPCR. E Detection of ECAR in colon cancer cells after knocking down c-MYC. F Detection of glucose content in colon cancer cells after knocking down c-MYC. G Detection of lactate production in colon cancer cells after knocking down c-MYC. H Detection of ATP/ADP in colon cancer cells after knocking down c-MYC. I RT-qPCR to detect the mRNA expression of GLUT1 and LDHA in colon cancer cells after knocking down c-MYC. The cell experiment was repeated three times. ${ }^{*} p<0.05$ between two groups.

curtailed glycolytic metabolism, thereby inhibiting the proliferation and chemoresistance of colon cancer cells.

\section{Knockdown of OLR1 suppresses glycolytic metabolism by downregulating C-MYC/SULT2B1 axis to restrain the proliferation and chemoresistance of colon cancer cells} Based on the above results, we speculated that OLR1 could activate the transcription of SULT2B1 by promoting C-MYC to enhance glycolytic metabolism, cell proliferation, and chemotherapy resistance. A cell line with $O L R 1$ knockdown and SULT2B1 overexpression was successfully constructed to further verify this conjecture (Fig. 6A). ECAR, glucose uptake, lactate production, ATP/ADP ratio, and GLUT1 and LDHA expression were evaluated, which depicted that overexpression of SULT2B1 nullified the repression of glycolysis induced by OLR1 knockdown (Fig. 6B-F). 


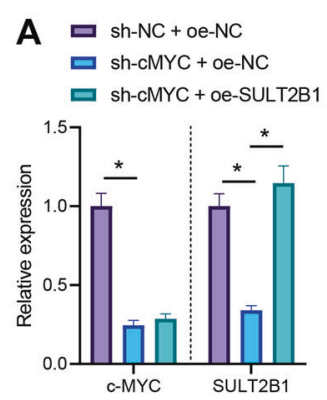

$$
\begin{aligned}
\text { B } & \rightarrow \text { sh-NC }+ \text { oe-NC } \\
& \quad \text { sh-CMYC + oe-NC } \\
& \neq \text { sh-CMYC + oe-SULT2B1 }
\end{aligned}
$$

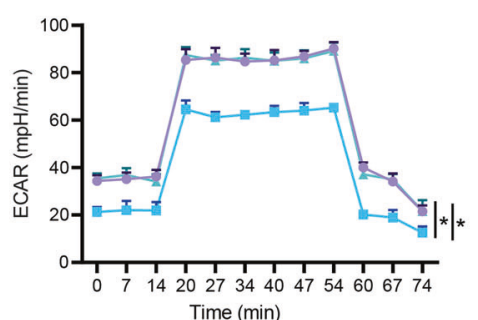

E $\mathrm{sh}-\mathrm{NC}+\mathrm{oe}-\mathrm{NC}$

口 sh-CMYC + oe-NC

- sh-CMYC + oe-SULT2B1

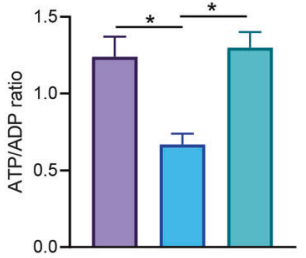

$J-s h-N C+o e-N C$

- sh-CMYC + oe-NC

- sh-CMYC + oe-SULT2B1

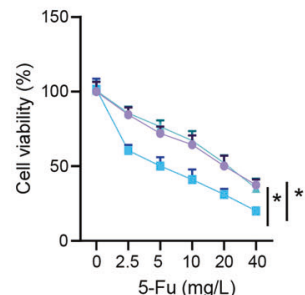

F

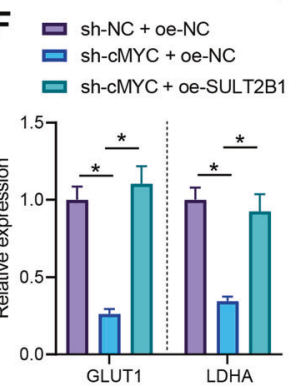

- sh-NC+ oe-NC

II- sh-CMYC + oe-NC

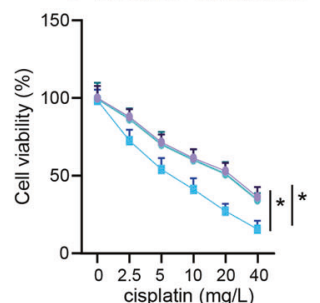

$\rightarrow$ sh-CMYC + oe-SULT2B1
G $\rightarrow$ sh-NC $+o e-N C$

-il- sh-CMYC + oe-NC

- Sh-CMYC + oe-SULT2B1

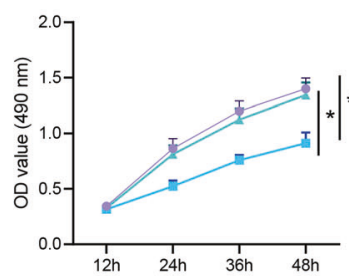

$$
\begin{aligned}
& \rightarrow \text { sh-NC + oe-NC } \\
& - \text { sh-cMYC + oe-NC } \\
& \rightarrow \text { sh-cMYC + oe-SULT2B1 }
\end{aligned}
$$

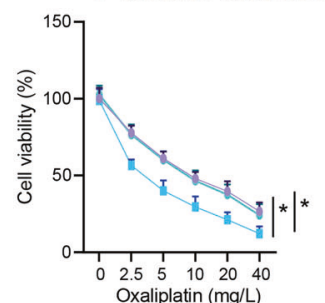

C $\square$ sh-NC + oe-NC

$\square$ sh-CMYC + oe-NC

$\square$ sh-CMYC + oe-SULT2B1

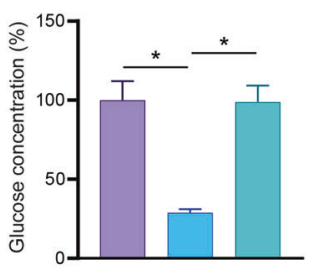

H $\square$ sh-NC + oe-NC

$\square$ sh-CMYC + oe-NC

- sh-cMYC + oe-SULT2B1

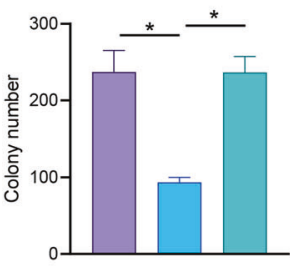

- sh-NC+ oe-NC

- sh-CMYC + oe-NC

$\rightarrow$ sh-CMYC + oe-SULT2B1

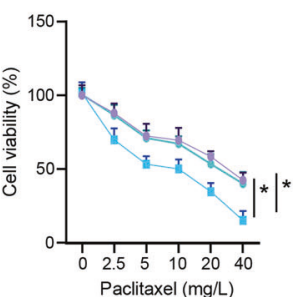

I $\square$ sh-NC + oe-NC

$\square$ sh-cMYC + oe-NC

$\square$ sh-CMYC + oe-SULT2B1

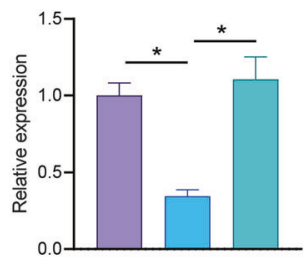

D 口h-NC + oe-NC

口 sh-CMYC + oe-NC

口 sh-CMYC + oe-SULT2B1

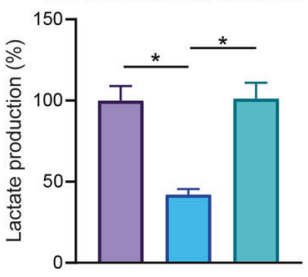

口 sh-NC + oe-NC

口 sh-CMYC + oe-NC

- sh-CMYC + oe-SULT2B1

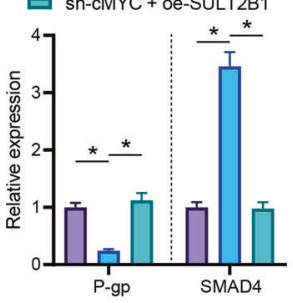

Fig. 5 Inactivation of c-MYC/SULT2B1 axis disturbs glycolytic metabolism to diminish proliferation and chemoresistance of colon cancer cells. Colon cancer cells were treated with sh-NC + oe-NC, sh-c-MYC + oe-NC, or sh-c-MYC + oe-SULT2B1. A The mRNA expression of c-MYC and SULT2B1 in colon cancer cells tested by RT-qPCR. B Detection of ECAR in colon cancer cells. C Glucose content in colon cancer cells. D Lactate production in colon cancer cells. E The ATP/ADP in colon cancer cells. F RT-qPCR to measure the mRNA expression of GLUT1 and LDHA in colon cancer cells. G MTS to calculate the proliferation level of colon cancer cells. H Colony formation assay to check the level of colony formation in colon cancer cells. I Detection of Ki67 mRNA expression in colon cancer cells by RT-qPCR. Colon cancer cells were treated with sh-NC + oe-NC, sh-c-MYC + oe-NC, or sh-c-MYC + oe-SULT2B1, followed by treatment with DDP, oxaliplatin, 5-Fu, and paclitaxel. J The drug resistance of colon cancer cells to DDP, oxaliplatin, 5-Fu, and paclitaxel detected by MTS. K RT-qPCR for detecting the mRNA expression of P-gp and SMAD4 in colon cancer cells. The cell experiments were repeated three times. ${ }^{*} p<0.05$ between two groups.

The MTS assay, clone formation assay, and Ki67 expression also manifested that overexpression of SULT2B1 annulled the suppression of the proliferation of colon cancer cells triggered by OLR1 knockdown (Fig. 6G-I). Colon cancer cells were stimulated by chemotherapeutic drugs (DDP, oxaliplatin, 5-Fu, and paclitaxel). It was observed that overexpression of SULT2B1 counteracted the sensitization effect of OLR1 knockdown to chemotherapy drugs (Fig. 6J, K). All in all, knockdown of OLR1 might downregulate SULT2B1 to repress glycolytic metabolism, thereby dampening the proliferation and chemoresistance of colon cancer cells.

\section{Knockdown of OLR1 blocks growth and chemoresistance of} colon cancer in nude mice by downregulating C-MYC/SULT2B1 In order to further explore whether OLR1/C-MYC/SULT2B1 axis could also modulate the growth and chemoresistance of colon cancer cells in vivo, a subcutaneous tumor transplantation experiment was implemented in nude mice. The LoVo cells transfected with sh-NC + oe-NC, sh-OLR1 + oe-NC, or sh-OLR1+ oe-SULT2B1 were subcutaneously injected into nude mice separately. As indicated by western blot analysis, expression of LOX-1, C-MYC, and SULT2B1 in tumor tissues of nude mice was lowered by knocking down OLR1, whilst SULT2B1 expression in tumor tissues of nude mice was markedly strengthened by oeSULT2B1 treatment in the presence of sh-OLR1As (Fig. 7A). Tumor growth rate and weight, and Ki67 expression were reduced in nude mice by sh-OLR1 treatment, which was negated by further oe-SULT2B1 treatment (Fig. 7B-D). These results suggested that knockdown of OLR1 slowed down the growth of colon cancer cells in nude mice via downregulation of SULT2B1.

The cells treated with sh-NC or sh-OLR1 were injected subcutaneously into nude mice to establish nude mice model of colon cancer. After intraperitoneal injection with drugs or normal saline, the tumor inhibition rate was detected. The result displayed that in the presence of oxaliplatin, the tumor inhibition rate was augmented by sh-OLR1 (Supplementary Table 4). Generally speaking, knockdown of OLR1 might inhibit the tumorigenicity and chemoresistance of colon cancer cells in nude mice by downregulating C-MYC and SULT2B1.

\section{DISCUSSION}

Patients with colon cancer are commonly diagnosed at regional or distant metastatic stage, which creates the need of adjuvant chemotherapy after their surgery or palliative chemotherapy for 

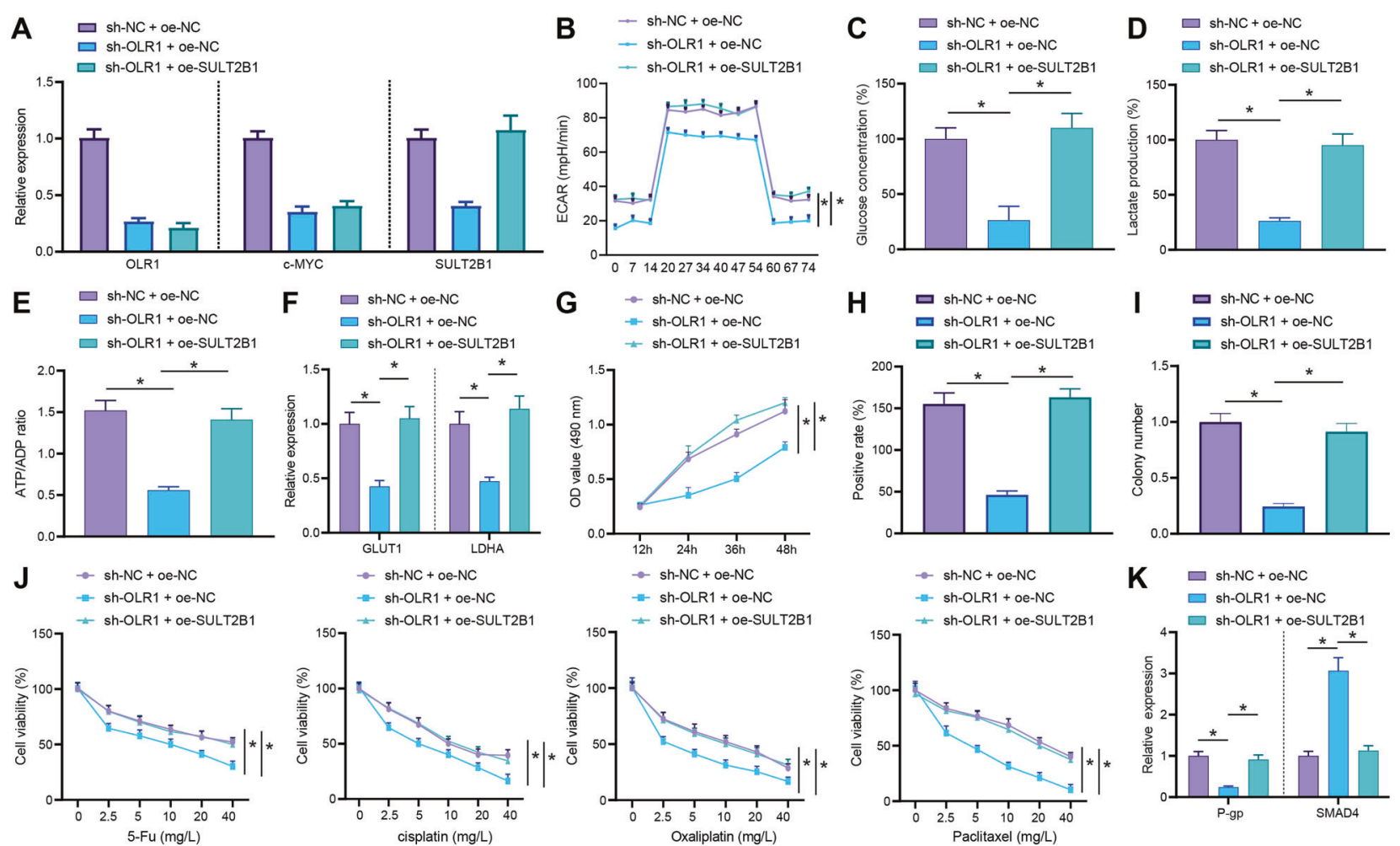

$\rightarrow-$ sh-NC + oe-NC

- sh-OLR1 + oe-NC
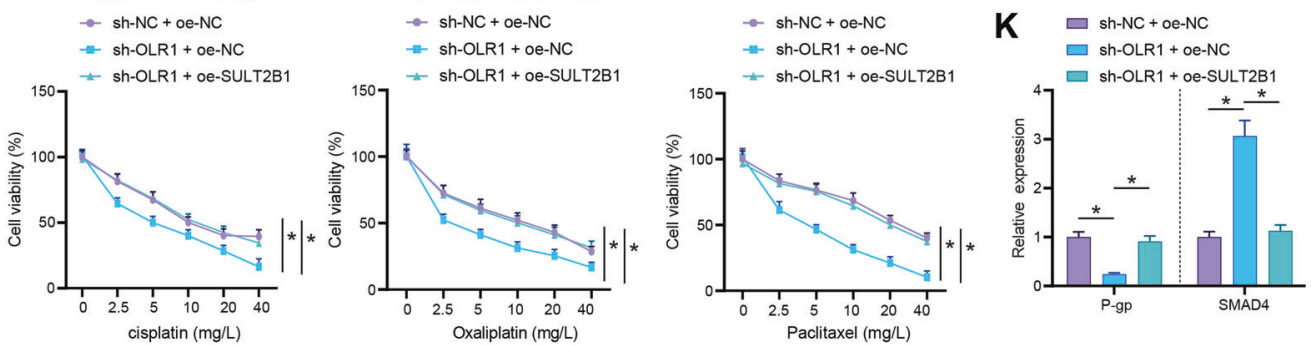

Fig. 6 Blockade of OLR1/C-MYC/SULT2B1 axis reduces glycolytic metabolism to restrain proliferation and chemoresistance of colon cancer cells. Colon cancer cells were transfected with sh-NC + oe-NC, sh-OLR1 + oe-NC, or sh-OLR1 + oe-SULT2B1. A The mRNA expression of OLR1, c-MYC, and SULT2B1 in colon cancer cells checked by RT-qPCR. B ECAR of colon cancer cells. C Glucose content in colon cancer cells. D Lactate production in colon cancer cells. E The ATP/ADP in colon cancer cells. F RT-qPCR to detect the mRNA expression of GLUT1 and LDHA in colon cancer cells. G The proliferation level of colon cancer cells estimated using MTS. $\mathbf{H}$ Detection of colon cancer cell clone formation. I The mRNA expression of Ki67 detected by RT-qPCR. Colon cancer cells were transfected with sh-NC + oe-NC, sh-OLR1 + oe-NC, or sh-OLR1 + oe-SULT2B1, followed by treatment with DDP, oxaliplatin, 5-Fu, and paclitaxel. J The drug resistance of colon cancer cells to DDP, oxaliplatin, 5-Fu, and paclitaxel assessed by MTS. $\mathbf{K}$ The expression of P-gp and SMAD4 in colon cancer cells tested by RT-qPCR. The cell experiment was repeated three times. ${ }^{*} p<0.05$ between two groups.

their metastatic disease [27]. However, the stemness and expansion abilities of colon cancer cells can result in resistance to conventional chemotherapies, which impacts the therapeutic effect of chemotherapy on patients with colon cancer [28]. Therefore, there exists an ongoing need to research the molecular mechanism behind the chemoresistance of colon cancer. In this context, we conducted this research to investigate the mechanism of OLR1/C-MYC/SULT2B1 axis in chemoresistance of colon cancer, and uncovered that knockdown of $O L R 1$ could downregulate c-MYC to diminish the transcription of SULT2B1, thus repressing glycolytic metabolism and then reducing the proliferation and chemoresistance of colon cancer cells (Fig. 8).

In the current work, evidence was obtained demonstrating that OLR1 was expressed at a high level in colon cancer tissues, shared negative correlation with the survival of patients with colon cancer, and facilitated glycolytic metabolism to promote proliferation and chemoresistance of colon cancer cells. A similar trend observed in the data of a prior research that OLR1 upregulation accelerated colon cancer onset, progression, and metastasis [8]. Moreover, Hirsch et al. has found that OLR1 is involved in maintaining the transformed state in developmentally diverse cancer cell lines and in tumor growth [29]. Of note, LOX-1 silencing contributes to impairment in proliferation rate, closing the scratch, growth, and tumorigenicity of CRC cells [30]. More importantly, a research conducted by Nakashima-Nakasuga et al. elucidated that CRC patients with high OLR1 expression in tumor tissues showed obviously poorer prognosis than individuals with low expression [31]. Moreover, the overexpression of $O L R 1$ has been detected in breast cancer tissues, and enhanced OLR1 expression is able to increase breast cancer cell proliferation [32]. Besides, a prior study noted that OLR1 presented with highexpression in osteosarcoma tissues and that osteosarcoma cell proliferation was accelerated when OLR1 was ectopically expressed [33]. It is widely reported that cancer cells commonly have augmented glycolytic metabolism for ATP generation, which frequently correlates to drug resistance in cancers [34]. Intriguingly, a prior work manifested that the induction of glycolytic metabolism facilitated chemoresistance in CRC [35]. Meanwhile, OLR1 has been identified to assume a crucial role in glycolytic metabolism [36]. Consistently, OLR1 upregulation was observed in pancreatic cancer tissues and was associated with the poor prognosis of patients, and then $O L R 1$ abrogation caused resistance of pancreatic cancer cells to gemcitabine [9]. Here, we propose that $O L R 1$ may participate in glycolytic metabolism, proliferation, and chemoresistance of colon cancer cells. In addition, our in vivo experiments manifested that OLR1 silencing diminished tumor weight and volume, Ki67 expression, and tumor chemoresistance in nude mice. Similarly, a prior research demonstrated that decreased Ki67 expression was correlated with reduced proliferation of CRC cells in vivo [37]. Of note, another research displayed that the inhibition of $O L R 1$ could prevent the tumorigenesis and metastasis formation of CRC in xenograft tumors of nude mice, suggesting OLR1 as a promising target for suppression of tumor progression and metastasis of CRC [38]. Partially concordant with our in vivo findings, the research of Xiong et al. ascertained that OLR1 upregulation caused by GSTM3TV2 was capable of augmenting chemoresistance in nude mice with subcutaneously injected pancreatic cancer cells and treated with gemcitabine [9]. 
A

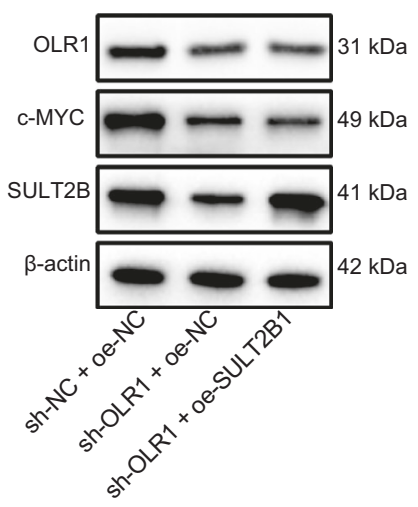

B

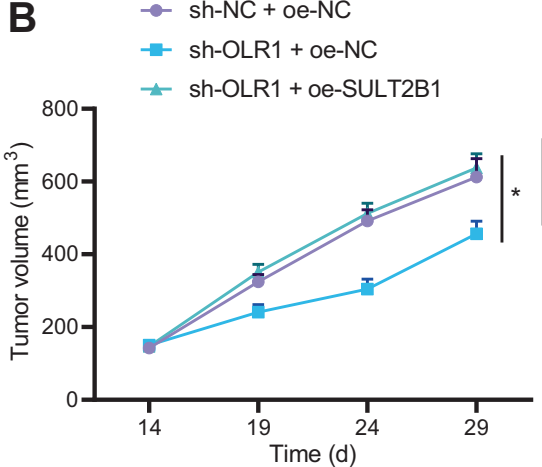

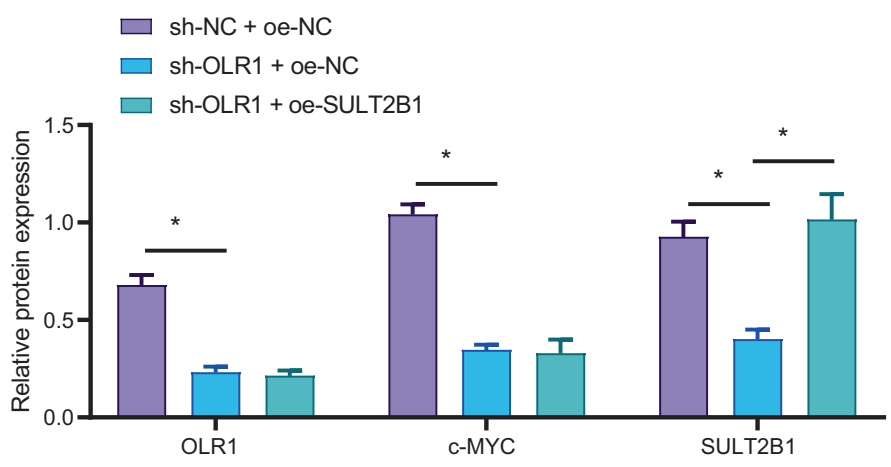

C

- sh-NC + oe-NC

- sh-OLR1 + oe-NC

A sh-OLR1 + oe-SULT2B1

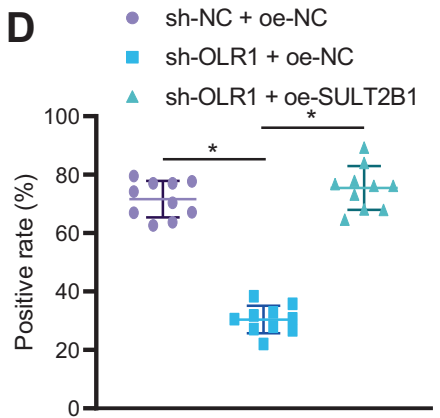

Fig. 7 The inactivation of OLR1/C-MYC/SULT2B1 axis restrains tumorigenicity and chemoresistance of colon cancer cells in nude mice. A The protein expression of LOX-1, c-MYC, and SULT2B1 detected by western blot analysis. B Growth curve of subcutaneous colon cancer xenograft in nude mice. C Weight of tumor in nude mice. D The expression of Ki67 in tumor tissues of nude mice detected by immunohistochemistry ( $n=10$ mice/group). ${ }^{*} p<0.05$.

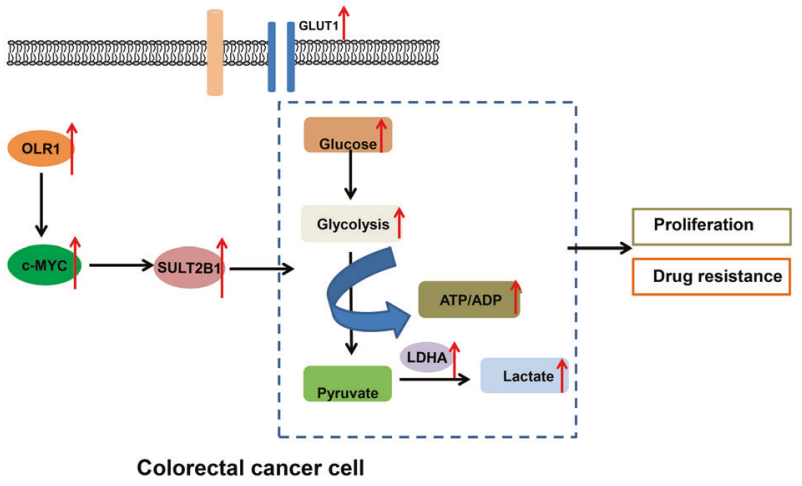

Fig. 8 Mechanism graph of the regulatory network and function of OLR1. OLR1 promotes colon cancer cell proliferation and chemoresistance by regulating C-MYC/SULT2B1 axis to enhance glycolysis metabolism.

As previously documented, OLR1 triggered c-MYC upregulation to accelerate pancreatic cancer metastasis [10]. A similar finding was noted in our work that OLR1 knockdown diminished c-MYC expression to suppress colon cancer cell glycolytic metabolism, proliferation, and chemoresistance. Corroborating findings were reported in a previous research that c-MYC was upregulated in CD133 colon cancer stem cells, and that upregulated c-MYC caused cell proliferation and chemoresistance of colon cancer [39]. In addition, c-MYC downregulation was reported to lower the proliferation and reduce glucose consumption, lactate production, and ATP production to depress glycolytic metabolism in DLD-1 and SW480 colon cancer cells, which was concordant with our results [40]. Also, stabled c-MYC was capable of contributing to
CRC cell proliferation by augmenting glycolytic metabolism [26]. However, the mechanism of c-MYC in glycolytic metabolism and chemoresistance in colon cancer remained to be further investigated. The obtained data in our research manifested that knockdown of c-MYC depressed the proliferation and chemoresistance of colon cancer cells by diminishing glycolytic metabolism via SULT2B1 downregulation. Concurrent with our results, a research conducted by $\mathrm{Li}$ et al. elaborated that SULT2B1 overexpression was linked to the development of CRC [13]. More importantly, SULT2B1 silencing caused disturbed HCC cell proliferation, which is partially in consent with our finding [41].

To sum up, the conclusion of the current study was that OLR1 knockdown disrupted the activation of c-MYC/SULT2B1 axis to repress glycolytic metabolism, thus restraining cell proliferation and chemoresistance in colon cancer. The result could open a novel candidate target in the field of colon cancer treatment. However, there exists limited research about the role of SULT2B1 in glycolytic metabolism and chemoresistance. This background calls for further research to figure out whether SULT2B1 manipulated glycolytic metabolism and chemoresistance in other tumors and find more convincible data of our study.

\section{DATA AVAILABILITY}

The datasets generated/analyzed during the current study are available from the corresponding author on reasonable request.

\section{REFERENCES}

1. Sharma SH, Thulasingam S, Nagarajan S. Terpenoids as anti-colon cancer agents - a comprehensive review on its mechanistic perspectives. Eur J Pharm. 2017;795:169-78.

2. Zhao $Y$, Hu X, Zuo X, Wang M. Chemopreventive effects of some popular phytochemicals on human colon cancer: a review. Food Funct. 2018;9:4548-68. 
3. Mehta A, Patel BM. Therapeutic opportunities in colon cancer: focus on phosphodiesterase inhibitors. Life Sci. 2019;230:150-61.

4. Aiello P, Sharghi M, Mansourkhani SM, Ardekan AP, Jouybari L, Daraei N, et al. Medicinal plants in the prevention and treatment of colon cancer. Oxid Med Cell Longev. 2019;2019:2075614.

5. Wang T, Ning K, Sun X, Zhang C, Jin LF, Hua D. Glycolysis is essential for chemoresistance induced by transient receptor potential channel $\mathrm{C} 5$ in colorectal cancer. BMC Cancer. 2018;18:207.

6. Grbcic P, Sedic M. Sphingosine 1-phosphate signaling and metabolism in chemoprevention and chemoresistance in colon cancer. Molecules. 2020;25:2436.

7. Nagelreiter IM, Parvardeh M, Narzt MS, Beer L, Kremslehner C, Muresan XM, et al. OLR1 scavenger receptor knockdown affects mitotic gene expression but is dispensable for oxidized phospholipid-mediated stress signaling in SZ 95 sebocytes. Mech Ageing Dev. 2018;172:35-44.

8. Murdocca M, De Masi C, Pucci S, Mango R, Novelli G, Di Natale C, et al. LOX-1 and cancer: an indissoluble liaison. Cancer Gene Ther. 2021. https://doi.org/10.1038/ s41417-020-00279-0. Epub ahead of print.

9. Xiong G, Liu C, Yang G, Feng M, Xu J, Zhao F, et al. Long noncoding RNA GSTM3TV2 upregulates LAT2 and OLR1 by competitively sponging let-7 to promote gemcitabine resistance in pancreatic cancer. J Hematol Oncol. 2019;12:97.

10. Yang G, Xiong G, Feng M, Zhao F, Qiu J, Liu Y, et al. OLR1 promotes pancreatic cancer metastasis via increased c-Myc expression and transcription of HMGA2. Mol Cancer Res. 2020;18:685-97.

11. Fatma H, Maurya SK, Siddique HR. Epigenetic modifications of c-MYC: role in cancer cell reprogramming, progression and chemoresistance. Semin Cancer Biol. 2020. https://doi.org/10.1016/j.semcancer.2020.11.008. Epub ahead of print.

12. Feng J, Ma J, Liu S, Wang J, Chen Y. A noncoding RNA LINC00504 interacts with c-Myc to regulate tumor metabolism in colon cancer. J Cell Biochem. 2019;120:14725-34.

13. Li S, Xie L, Du M, Xu K, Zhu L, Chu H, et al. Association study of genetic variants in estrogen metabolic pathway genes and colorectal cancer risk and survival. Arch Toxicol. 2018;92:1991-9.

14. Benson AB, Venook AP, Al-Hawary MM, Cederquist L, Chen YJ, Ciombor KK, et al. NCCN guidelines insights: colon cancer, version 2.2018. J Natl Compr Canc Netw. 2018;16:359-69.

15. Livak KJ, Schmittgen TD. Analysis of relative gene expression data using real-time quantitative PCR and the $2-\Delta \Delta C_{\mathrm{T}}$ method. Methods 2001;25:402-8

16. Lu W, Wang J, Yang G, Yu N, Huang Z, Xu H, et al. Posttranscriptional regulation of galectin-3 by miR-128 contributes to colorectal cancer progression. Oncotarget 2017;8:15242-51.

17. Xiong W, Ren ZG, Qiu SJ, Sun HC, Wang L, Liu BB, et al. Residual hepatocellular carcinoma after oxaliplatin treatment has increased metastatic potential in a nude mouse model and is attenuated by Songyou Yin. BMC Cancer. 2010;10:219.

18. Guo P, Wang J, Gao W, Liu X, Wu S, Wan B, et al. Salvianolic acid B reverses multidrug resistance in nude mice bearing human colon cancer stem cells. Mol Med Rep. 2018;18:1323-34.

19. Ma J, Gao G, Lu H, Fang D, Li L, Wei G, et al. Reversal effect of ginsenoside Rh2 on oxaliplatin-resistant colon cancer cells and its mechanism. Exp Ther Med. 2019;18:630-6.

20. Fu J, Weise AM, Falany JL, Falany CN, Thibodeau BJ, Miller FR, et al. Expression of estrogenicity genes in a lineage cell culture model of human breast cancer progression. Breast Cancer Res Treat. 2010;120:35-45.

21. Gonthier K, Poluri RTK, Audet-Walsh E. Functional genomic studies reveal the androgen receptor as a master regulator of cellular energy metabolism in prostate cancer. J Steroid Biochem Mol Biol. 2019;191:105367.

22. Sabbir MG. Progesterone induced Warburg effect in HEK293 cells is associated with post-translational modifications and proteasomal degradation of progesterone receptor membrane component 1. J Steroid Biochem Mol Biol. 2019;191:105376.

23. Wang WL, Jiang ZR, Hu C, Chen $C$, Hu ZQ, Wang AL, et al. Pharmacologically inhibiting phosphoglycerate kinase 1 for glioma with NG52. Acta Pharm Sin. 2021;42:633-40.

24. Qi J, Zhou N, Li L, Mo S, Zhou Y, Deng $Y$, et al. Ciclopirox activates PERKdependent endoplasmic reticulum stress to drive cell death in colorectal cancer. Cell Death Dis. 2020;11:582.

25. Lin CX, Tu CW, Ma YK, Ye PC, Shao X, Yang ZA, et al. Nobiletin inhibits cell growth through restraining aerobic glycolysis via PKA-CREB pathway in oral squamous cell carcinoma. Food Sci Nutr. 2020;8:3515-24.

26. Tang J, Yan T, Bao Y, Shen C, Yu C, Zhu X, et al. LncRNA GLCC1 promotes colorectal carcinogenesis and glucose metabolism by stabilizing c-Myc. Nat Commun. 2019;10:3499.

27. Wu C. Systemic therapy for colon cancer. Surg Oncol Clin N. Am. 2018;27:235-42.

28. Chen S, Tang Y, Liu Y, Zhang P, Lv L, Zhang X, et al. Exosomes derived from miR375-overexpressing human adipose mesenchymal stem cells promote bone regeneration. Cell Prolif. 2019;52:e12669.
29. Hirsch HA, lliopoulos D, Joshi A, Zhang $Y$, Jaeger SA, Bulyk M, et al. A transcriptional signature and common gene networks link cancer with lipid metabolism and diverse human diseases. Cancer Cell. 2010;17:348-61.

30. Murdocca M, Mango R, Pucci S, Biocca S, Testa B, Capuano R, et al. The lectin-like oxidized LDL receptor-1: a new potential molecular target in colorectal cancer. Oncotarget 2016;7:14765-80.

31. Nakashima-Nakasuga C, Hazama S, Suzuki N, Nakagami Y, Xu M, Yoshida S, et al. Serum LOX-1 is a novel prognostic biomarker of colorectal cancer. Int J Clin Oncol. 2020;25:1308-17.

32. Wang $B$, Zhao $H$, Zhao $L$, Zhang $Y$, Wan $Q$, Shen $Y$, et al. Up-regulation of OLR1 expression by TBC1D3 through activation of TNFa/NF-KB pathway promotes the migration of human breast cancer cells. Cancer Lett. 2017;408:60-70.

33. Jiang L, Jiang $S$, Zhou W, Huang J, Lin $Y$, Long $H$, et al. Oxidized low density lipoprotein receptor 1 promotes lung metastases of osteosarcomas through regulating the epithelial-mesenchymal transition. J Transl Med. 2019;17:369.

34. Ma L, Zong X. Metabolic symbiosis in chemoresistance: refocusing the role of aerobic glycolysis. Front Oncol. 2020;10:5.

35. Wang $X$, Zhang $H$, Yang $H$, Bai $M$, Ning T, Deng $T$, et al. Exosome-delivered circRNA promotes glycolysis to induce chemoresistance through the miR-122PKM2 axis in colorectal cancer. Mol Oncol. 2020;14:539-55.

36. Xing $\mathrm{K}$, Wang $\mathrm{K}$, Ao $\mathrm{H}$, Chen $\mathrm{S}$, Tan $\mathrm{Z}$, Wang $\mathrm{Y}$, et al. Comparative adipose transcriptome analysis digs out genes related to fat deposition in two pig breeds. Sci Rep. 2019;9:12925.

37. Lee MS, Helms TL, Feng N, Gay J, Chang QE, Tian F, et al. Efficacy of the combination of MEK and CDK4/6 inhibitors in vitro and in vivo in KRAS mutant colorectal cancer models. Oncotarget 2016;7:39595-608.

38. Murdocca M, Capuano R, Pucci S, Cicconi R, Polidoro C, Catini A, et al. Targeting LOX1 inhibits colorectal cancer metastasis in an animal model. Front Oncol. 2019;9:927.

39. Zhang HL, Wang P, Lu MZ, Zhang SD, Zheng L. c-Myc maintains the self-renewal and chemoresistance properties of colon cancer stem cells. Oncol Lett. 2019;17:4487-93.

40. He T, Zhou H, Li C, Chen Y, Chen X, Li C, et al. Methylglyoxal suppresses human colon cancer cell lines and tumor growth in a mouse model by impairing glycolytic metabolism of cancer cells associated with down-regulation of c-Myc expression. Cancer Biol Ther. 2016;17:955-65.

41. Wang ZY, Zhu Z, Wang HF, Qin B, Liu J, Yao XH, et al. Downregulation of cirCDYNC1H1 exhibits inhibitor effect on cell proliferation and migration in hepatocellular carcinoma through miR-140-5p. J Cell Physiol. 2019;234:17775-85.

\section{AUTHOR CONTRIBUTIONS}

T.Z. and Y.L. wrote the paper; K.S. and Q.W. conceived the experiments; J.Z. analyzed the data; T.Z. collected and provided the sample for this study. All authors have read and approved the final submitted manuscript.

\section{COMPETING INTERESTS}

The authors declare no competing interests.

\section{ETHICS APPROVAL AND CONSENT TO PARTICIPATE}

All participants from China-Japan Union Hospital of Jilin University or their guardians provided signed informed consent prior to enrollment. The Ethics Committee of China-Japan Union Hospital of Jilin University provided Ethical Approval for the experiments involving human being in this study, which conformed to the Declaration of Helsinki. Animal experiments were ratified by Animal Ethics Committee of China-Japan Union Hospital of Jilin University in accordance with the animal ethics standards expressed in the Care and Use of Laboratory Animals published by the US National Institutes of Health. Extensive efforts were made to minimize suffering of the included animals.

\section{ADDITIONAL INFORMATION}

Supplementary information The online version contains supplementary material available at https://doi.org/10.1038/s41419-021-04174-w.

Correspondence and requests for materials should be addressed to Jiayu Zhang.

Reprints and permission information is available at http://www.nature.com/ reprints

Publisher's note Springer Nature remains neutral with regard to jurisdictional claims in published maps and institutional affiliations. 
(c) (i) Open Access This article is licensed under a Creative Commons cc) Attribution 4.0 International License, which permits use, sharing, adaptation, distribution and reproduction in any medium or format, as long as you give appropriate credit to the original author(s) and the source, provide a link to the Creative Commons license, and indicate if changes were made. The images or other third party material in this article are included in the article's Creative Commons license, unless indicated otherwise in a credit line to the material. If material is not included in the article's Creative Commons license and your intended use is not permitted by statutory regulation or exceeds the permitted use, you will need to obtain permission directly from the copyright holder. To view a copy of this license, visit http://creativecommons. org/licenses/by/4.0/.

(c) The Author(s) 2021 